\title{
Adaptação Acadêmica e Coping em Estudantes Universitários
}

\author{
Rodrigo Carvalho Carlotto - Universidade Federal de Santa Maria, Santa Maria, Brasil \\ Marco Antônio Pereira Teixeira - Universidade Federal do Rio Grande do Sul, Porto Alegre, Brasil \\ Ana Cristina Garcia Dias - Universidade Federal do Rio Grande do Sul, Porto Alegre, Brasil
}

\begin{abstract}
Resumo
Este estudo investigou as possíveis relações entre estratégias de coping e a adaptação acadêmica em estudantes universitários. Além disso, procurou-se verificar se o gênero ou a etapa do curso (primeiro ou último ano) influenciariam na escolha de determinadas estratégias e nos níveis de adaptação ao ensino superior. Participaram desse estudo 412 acadêmicos. Para a coleta dos dados utilizou-se o Questionário de Vivências Acadêmicas - Versão Reduzida (QVA-r), a Escala Modos de Enfrentamento de Problemas (EMEP) e um questionário de dados sociodemográficos. Pode-se perceber que as estratégias focadas no problema e no suporte social correlacionaram-se positivamente com o processo de adaptação acadêmica e, na emoção e nas práticas religiosas/pensamento fantasioso, por sua vez, estiveram relacionadas negativamente com o processo de ajustamento ao ensino superior.

Palavras-chave: adaptação à universidade, coping, vivências acadêmicas, ensino superior, universitários
\end{abstract}

Adaptation to University and Coping among Undergraduate Students

\begin{abstract}
This study investigated possible correlations between coping strategies and academic adaptation among undergraduate students. Furthermore, we sought to verify whether students' gender and college year (first or last year) would influence their choice of specific strategies as well as their levels of adaptation to higher education. The sample consisted of 412 undergraduate students. The instruments used were the Academic Experiences Questionnaire - Reduced Version (AEQ-r), Ways of Coping Questionnaire (WAYS) and a sociodemographic questionnaire. Results revealed that strategies focused both on the problem and social support had a positive correlation with academic adaptation. On the other hand, strategies focused on emotion and religious practices/fanciful thinking were negatively related to the process of adaptation to higher education.

Keywords: adaptation to university, coping, academic experiences, higher education, undergraduate students
\end{abstract}

\section{Adaptación Académica y Coping en Estudiantes Universitarios}

\begin{abstract}
Resumen
Este estudio investigó las posibles relaciones entre las estrategias de coping y la adaptación académica en estudiantes universitarios. Además, buscó verificar si el género o la etapa del curso (primer o último año) influyeron en la elección de determinadas estrategias y en los niveles de adaptación a la enseñanza superior. Participaron del estudio 412 académicos y para la cosecha de datos se utilizó el Cuestionario de Vivencias Académicas - versión reducida - (CVA-r), la Escala Maneras de Enfrentamiento de Problemas (EMEP) y un cuestionario de datos sociodemográficos. Se pudo percibir que las estrategias enfocadas en el problema y en el apoyo social se correlacionaron positivamente con el proceso de adaptación académica. Las estrategias enfocadas en la emoción y en las prácticas religiosas/pensamiento fantasioso, a su vez, estuvieron relacionadas negativamente con el proceso de ajuste en la enseñanza superior.

Palabras clave: adaptación a la universidad, coping, vivencias académicas, educación superior, universitarios
\end{abstract}

A trajetória universitária promove significativas mudanças na vida dos jovens (Almeida, Guisande, Soares, \& Saavedra, 2006). Especialmente nos anos iniciais, os estudantes enfrentarão uma série de desafios associados às dimensões acadêmica, social, pessoal, institucional e vocacional. Nos âmbitos acadêmico e institucional, dificuldades relacionadas ao novo ritmo de estudo, ao sistema de avaliação e as regras burocráticas da universidade podem se constituir em importantes estressores para os universitários. Da mesma forma, aspectos pessoais e vocacionais como o desenvolvimento de uma identidade profissional e o comprometimento com a formação acadêmica representarão importantes desafios para os estudantes. Além desses elementos, questões relacionadas à dimensão social, como os novos padrões de relacionamento com a família, professores, colegas e figuras de autoridade também podem interferir no processo de adaptação ao ensino superior (Almeida \& Soares, 2003).

Diante desse contexto potencialmente estressor, vários estudos nacionais (Guerreiro-Casanova \& Polydoro, 2010; Igue, Bariani, \& Milanesi, 2008; Soares, Poubel \& Mello, 2009; Teixeira, Dias, Wottrich \& Oliveira, 2008) e internacionais (Almeida et al., 2006; Diniz \& Almeida, 2006; Swenson, Nordstrom, \& Hiester 2008) têm investigado os fatores que podem atuar 
de forma positiva ou negativa durante a integração do estudante na universidade. De forma geral, nessas pesquisas a adaptação à universidade tem sido entendida como uma mudança normal para os jovens que seguem esse caminho, constituindo-se em um processo multidimensional que envolve aspectos institucionais, relacionais, vocacionais, pessoais, entre outros (Pascarella \& Terenzini, 2005).

As estratégias de enfrentamento que os estudantes utilizam para lidar com situações estressoras e como essas estratégias contribuem para a adaptação acadêmica tem sido um tema investigado no contexto da integração ao ensino superior (Dyson \& Renk, 2006). Essas estratégias de enfrentamento (ou simplesmente coping) referem-se aos recursos emocionais, cognitivos e comportamentais utilizados pelos indivíduos na tentativa de lidar com as situações estressoras (Lazarus \& Folkman, 1984). Ao perceber uma situação como estressante, as pessoas costumam experimentar uma sensação emocional desagradável e tendem a adotar estratégias que permitam minimizar o seu sofrimento (Dyson \& Renk, 2006).

$\mathrm{Na}$ literatura internacional, um bom número de estudos tem investigado as relações entre coping e adaptação acadêmica (Aspinwall \& Taylor, 1992; Cabral \& Matos, 2010; Costa \& Leal, 2006; Feenstra, Banyard, Rine, \& Hopkins, 2001). Em geral, observa-se que as estratégias ativas, direcionadas à resolução de problemas têm apresentado correlação direta e positiva com o ajuste à universidade (Aspinwall \& Taylor, 1992, Cabral \& Mattos, 2010). Do mesmo modo, tem-se constatado que o uso de estratégias baseadas no suporte social, como a busca de apoio em amigos (Costa \& Leal, 2006) e na família (Feenstra et al., 2001), constituem-se em bons preditores da adaptação acadêmica. Em contrapartida, comportamentos essencialmente focados na emoção, como o uso da evitação e da esquiva, têm sido relacionados a índices inferiores de ajustamento ao ensino superior (Aspinwall \& Taylor, 1992).

Ainda em âmbito internacional, estudos desenvolvidos junto ao público universitário têm apontado que questões relacionadas ao gênero e ao ano em que o acadêmico se encontra poderiam influenciar na escolha de determinadas estratégias de enfrentamento (Bardagi \& Hutz, 2011). De modo geral, constata-se que as estudantes do sexo feminino costumam usar com mais frequência estratégias baseadas na emoção (Matud, 2004) e no apoio social (Costa \& Leal, 2006). Já entre os homens, haveria uma maior tendência na utilização de estratégias focadas no problema (Dyson \& Renk,
2006; Lazarus \& Folkman, 1984; Matud, 2004). Quanto ao ano, tem-se observado que calouros utilizariam com mais frequência estratégias de distanciamento, de evitação e isolamento, fazendo menor uso de atitudes baseadas no suporte social. Por sua vez, formandos usariam com mais frequência técnicas ativas de resolução de problemas (Toray \& Cooley, 1998).

No Brasil, os estudos sobre coping concentram-se, principalmente, no ambiente ocupacional (Dela Coleta \& Dela Coleta, 2008; Suehiro, Santos, Hatamoto, \& Cardoso, 2008) e nas áreas relacionadas à saúde (Adolhe, Guido, \& Bianchi, 2009; Neme \& Lipp, 2010). No entanto, já existem algumas publicações que se propõe investigar as estratégias de enfrentamento e suas consequências junto ao meio acadêmico (Bardagi \& Hutz, 2011). De forma geral, os estudos desenvolvidos com o público universitário têm procurado descrever as possíveis relações entre o coping e variáveis de saúde psíquica, como estresse (Santos \& Alves Júnior, 2007) e depressão (Coelho, Albuquerque, Martins, D’Albuquerque, \& Neves, 2008).

É fato que o coping pode desempenhar um papel importante na adaptação dos estudantes. No entanto, observa-se que, no território nacional, as pesquisas sobre o impacto dessas estratégias no processo de ajustamento ao ensino superior ainda são escassas. Nesse sentido, com o intuito de colaborar para o desenvolvimento de estudos na área, buscou-se investigar as possíveis relações entre estratégias de coping e o processo de adaptação acadêmica em estudantes universitários. Além disso, procurou-se verificar se as questões de gênero, ou o fato do aluno estar no primeiro ou último ano de seu curso influenciariam na escolha de determinadas estratégias e nos níveis de adaptação ao ensino superior.

\section{Método}

\section{Participantes}

Participaram desse estudo 412 estudantes universitários de primeiro e último ano de uma Universidade Pública e de um Instituto Federal de Educação do interior do Rio Grande do Sul. A amostra foi constituída por 258 mulheres $(62,6 \%)$ e 154 homens (37,4\%). Desses, $240(58,3 \%)$ frequentavam o ano inicial e 172 $(41,7 \%)$, o ano final dos seguintes cursos superiores: Medicina Veterinária (15,0\%), Ciências Contábeis $(12,9 \%)$, Educação Especial (12,4\%), Fonoaudiologia (9,2\%), Administração (9,2\%), Enfermagem (7,8\%), Produção de Grãos (6,6\%), Artes Cênicas (5,8\%), Sistemas da Informação (5,8\%), Ciências Biológicas (5,6\%), 
Agronegócio (5,6\%) e Matemática (5,1\%). A idade dos estudantes universitários variou de 16 a 43 anos, sendo a média 23,00 e o desvio padrão 4,75 anos. A amostra foi escolhida por conveniência, sendo que os critérios de inclusão adotados foram: (1) estar frequentando regularmente o primeiro ou o último ano de cursos de graduação das instituições pesquisadas; e (2) responder de forma voluntária e imediata o questionário. Os alunos de outros anos letivos que porventura estivessem frequentando disciplinas isoladas nas turmas pesquisadas foram excluídos do estudo. Inicialmente, os dados foram coletados junto a 504 estudantes, mas devido a respostas faltantes e casos extremos apenas 412 foram incluídos nas análises.

\section{Instrumentos e Procedimentos}

Os aspectos sociodemográficos e as variáveis coping e adaptação foram medidas por meio de instrumentos específicos, descritos a seguir:

Questionário de Caracterização da Amostra: Para avaliar os aspectos sociodemográficos da população, foi utilizado um questionário de caracterização da amostra desenvolvido especialmente para o estudo.

Questionário de Vivências Acadêmicas - versão reduzida (QVA-r): Adaptado para o Brasil por Granado, Santos, Almeida, Soares e Guisande (2005), o instrumento avalia cinco grandes áreas de vivências no âmbito universitário: pessoal (ajustamento psicológico e bem-estar geral, com foco na ausência de características associadas à depressão e ansiedade), interpessoal (nível de integração com amigos e percepção de apoio), carreira (decisão e satisfação com a escolha), estudo (nível de organização e compromisso com estudo) e institucional (satisfação e vínculo com a instituição). $\mathrm{O}$ QVA-r, no estudo de adaptação, apresentou evidências de validade de construto por análise fatorial e índices de consistência interna nas subescalas entre 0,77 e 0,86 . Conforme os autores, é possível utilizar uma combinação das escalas como uma medida global de adaptação acadêmica (Granado et al., 2005). Os itens são respondidos em uma escala Likert de cinco pontos, e nesta pesquisa, os escores de cada subescala foram computados de modo que o escore mínimo possível seja um e o máximo cinco.

Escala Modos de Enfrentamento de Problemas (EMEP): Trata-se de uma escala composta por itens que abordam pensamentos e ações utilizados durante o enfrentamento de estressores específicos. A versão adaptada (Gimenez \& Queiroz, 1997) e validada (Seidl, Tróccoli, \& Zannon, 2001) para a população brasileira identifica quatro modos de enfrentamento/ coping: estratégias focadas no problema, estratégias focadas na emoção, práticas religiosas/pensamento fantasioso e busca de suporte social. A EMEP, no estudo de Seidl et al. (2001), apresentou evidências de validade de construto por análise fatorial e índices de consistência interna nas subescalas entre 0,70 e 0,84. Do mesmo modo que o QVA-r, os itens são respondidos em uma escala Likert de cinco pontos e, nesta pesquisa, os escores de cada subescala foram computados de forma que o escore mínimo possível seja um e o máximo cinco.

Após aprovação pelo Comitê de Ética em Pesquisa da Universidade Federal de Santa Maria, o projeto foi apresentado às coordenações dos cursos pesquisados nas duas instituições de ensino superior. Após a autorização, foram indicados os dias e horários mais adequados para a realização da coleta junto às turmas. O questionário anônimo foi respondido coletivamente em sala de aula, sendo que nenhum jovem recusou-se a participar. Em média, o tempo de aplicação dos instrumentos foi de 30 minutos.

\section{Análise dos Dados}

Inicialmente foi realizada uma análise preliminar para identificar casos com dados faltantes ou com escores extremos nas variáveis centrais da pesquisa (vivências acadêmicas e coping). Foram excluídos casos que tivessem dados faltantes ou escores padronizados superiores a 3 (em módulo) em quaisquer das subescalas do QVA-r e da EMEP, o que resultou na eliminação de 92 casos (totalizando 412 casos analisados). Para verificar as possíveis relações entre coping e adaptação acadêmica foram utilizadas análises correlacionais (Pearson) e de regressão linear. Posteriormente, efetuaram-se comparações de médias entre grupos relevantes (homens e mulheres, calouros e formandos) por meio de análises de covariância multivariadas.

\section{Resultados}

A Tabela 1 apresenta as médias e os desvios-padrão obtidos para as variáveis de adaptação à universidade e coping, os índices de fidedignidade dos instrumentos, e as correlações entre as variáveis. Pode-se constatar que os instrumentos apresentaram estimativas de fidedignidade razoáveis, exceto pela subescala EMEP-Práticas Religiosas/Pensamento Fantasioso, cujo coeficiente alfa ficou ligeiramente abaixo do desejável $(0,70)$. Quanto às médias nas subescalas de coping, observou-se que as estratégias focadas no problema $(M=3,66)$ 
foram as mais utilizadas; em contrapartida, as estratégias baseadas na emoção $(M=2,53)$ obtiveram as menores médias. Já em relação ao QVA-r, observa-se que os valores obtidos nesse instrumento foram relativamente elevados, situando-se entre 3 e 4, sendo 3 o ponto médio da escala.

Observou-se que as quatro subescalas da EMEP (Problema, Emoção, Suporte Social e Práticas Religiosas/Pensamento Fantasioso) apresentaram correlações estatisticamente significativas com a maior parte das dimensões do QVA-r. Apenas não foram verificadas correlações estatisticamente significativas entre a subescala EMEP-Práticas Religiosas/Pensamento Fantasioso e as dimensões QVA-r Interpessoal e QVA-r Institucional, e entre a escala EMEP-Emoção e QVA-r Estudo.

Pode-se constatar que a subescala EMEP-Problema correlacionou-se positivamente com todas as dimensões do QVA-r. Em especial, vale destacar a relação entre EMEP-Problema e QVA-r Estudo $(0,40)$. Por outro lado, verificou-se que a subescala EMEP-Emoção esteve associada a níveis inferiores de adaptação acadêmica. Destaca-se a correlação apresentada entre as subescalas EMEP-Emoção e QVA-r Pessoal $(-0,52)$. A subescala EMEP-Suporte Social mostrou-se positivamente relacionada com as dimensões QVA-r Interpessoal $(0,27)$, Estudo $(0,15)$, Institucional $(0,12)$ e Carreira $(0,08)$, e negativamente relacionada com a dimensão QVA-r Pessoal $(-0,15)$. Também foram observadas correlações positivas entre a subescala EMEP-Práticas Religiosas/Pensamento Fantasioso e a dimensão QVA-r Estudo $(0,17)$, e negativas com as dimensões QVA-r Pessoal $(-0,33)$ e Carreira $(-0,10)$.

Com o intuito de determinar o potencial preditivo das estratégias de coping para a adaptação acadêmica, realizou-se uma análise de regressão linear múltipla, cujos resultados são exibidos na Tabela 2 .

A variável dependente da análise de regressão múltipla foi um escore geral do instrumento QVA-r. Pode-se observar que todas as subescalas do instrumento QVA-r se mostraram positivamente correlacionadas. Assim, foi feita uma análise de componentes principais, a fim de criar um índice geral de vivências acadêmicas, a partir

Tabela 1

Dados Descritivos e Correlações entre as Escalas de Adaptação Acadêmica e Coping

\begin{tabular}{|c|c|c|c|c|c|c|c|c|c|c|}
\hline & $\begin{array}{c}\mathrm{M} \\
(\mathrm{DP}) \\
\end{array}$ & Alfa & 1 & 2 & 3 & 4 & 5 & 6 & 7 & 8 \\
\hline 1. QVA-r & 3,64 & 0,86 & & & & & & & & \\
\hline Pessoal & $(0,70)$ & & & & & & & & & \\
\hline 2. QVA-r & 3,84 & 0,86 & 0,24 & & & & & & & \\
\hline Interpessoal & $(0,61)$ & & & & & & & & & \\
\hline 3. QVA-r & 3,95 & 0,89 & 0,32 & 0,25 & & & & & & \\
\hline Carreira & $(0,66)$ & & & & & & & & & \\
\hline 4. QVA-r & 3,62 & 0,77 & 0,22 & 0,16 & 0,26 & & & & & \\
\hline Estudo & $(0,59)$ & & & & & & & & & \\
\hline 5. QVA-r & 3,98 & 0,70 & 0,27 & 0,28 & 0,38 & 0,26 & & & & \\
\hline Institucional & $(0,55)$ & & & & & & & & & \\
\hline 6. EMEP & 3,66 & 0,86 & 0,21 & 0,18 & 0,26 & 0,40 & 0,26 & & & \\
\hline Problema & $(0,49)$ & & & & & & & & & \\
\hline 7. EMEP & 2,53 & 0,79 & $-0,52$ & $-0,13$ & $-0,11$ & $-0,07$ & $-0,12$ & 0,01 & & \\
\hline Emoção & $(0,57)$ & & & & & & & & & \\
\hline 8. EMEP & 2,96 & 0,69 & $-0,33$ & 0,04 & $-0,10$ & 0,17 & 0,06 & 0,22 & 0,42 & \\
\hline Religião/Fantasia & $(0,75)$ & & & & & & & & & \\
\hline 9. EMEP & 3,26 & 0,76 & $-0,15$ & 0,27 & 0,08 & 0,15 & 0,12 & 0,24 & 0,19 & 0,37 \\
\hline Suporte Social & $(0,89)$ & & & & & & & & & \\
\hline
\end{tabular}

Nota. QVA-r = Questionário de Vivências Acadêmicas-revisado, EMEP = Escala Modos de Enfrentamento de Problemas. Para correlações iguais ou superiores a $0,12, p<0,01$; correlação entre 0,08 e $0,11, p<0,05$; correlações inferiores a $0,08, p>0,05$. 
dos escores brutos das subescalas Pessoal, Interpessoal, Carreira, Estudo e Institucional. Os resultados mostraram o primeiro componente explicando $41,4 \%$ da variância total das subescalas, com todas as subescalas com cargas acima de 0,60 nesse componente. Assim, foram salvos os escores fatoriais resultantes (pelo método de regressão), constituindo um índice, para cada indivíduo, das diversas vivências acadêmicas avaliadas pelo instrumento QVA-r. Uma inspeção visual da distribuição dessa variável, assim como de seus valores de assimetria $(-0,32)$ e curtose $(-0,06)$, indicou tratar-se de uma distribuição normal.

As variáveis independentes da análise de regressão foram as subescalas do instrumento EMEP. A análise de regressão mostrou que o modelo foi significativo, $F(4,407)=36,21, p<0,001$, havendo poucos resíduos autocorrelacionados (índice de Durbin-Watson com valor de 2,10, ou seja, muito próximo do ideal de $2,00)$. O $R^{2}$ ajustado apontou que o conjunto das variáveis independentes explicou $25,5 \%$ da variância total do índice de vivências acadêmicas. Análises complementares mostraram a inexistência de casos com resíduos padronizados superiores a três, bem como a homocedasticidade e normalidade dos resíduos, indicando a adequação dos dados para a análise.

Os betas são interpretados de maneira semelhante aos coeficientes de correlação da Tabela 1 , ou seja, valores positivos indicam relacionamento linear positivo entre a variável independente e a variável dependente (vivências acadêmicas), e vice-versa. Observou-se que, com exceção da estratégia de coping religioso, as outras três estratégias de coping se apresentaram estatisticamente relacionadas ao índice geral de vivências acadêmicas. Em especial, constatou-se que a variável EMEP-Problema foi a mais fortemente relacionada com as vivências acadêmicas (que indicam adaptação ao ambiente acadêmico).

Por fim, na Tabela 3 apresentam-se os resultados obtidos por meio das comparações de médias entre grupos relevantes (calouros e formandos, homens e mulheres). As comparações de médias foram conduzidas com duas análises de covariância multivariadas (MANCOVAS), uma para o conjunto de variáveis de vivências acadêmicas (QVA-r) e outra para as variáveis de coping (EMEP), tendo como covariável a idade. Os fatores independentes foram sexo (masculino ou feminino) e etapa do curso (ingressante ou concluinte). A idade foi incluída como covariável, pois a maturidade promovida pela experiência de vida pode ser um fator que contribui para a adaptação a diferentes contextos e o enfrentamento de situações desafiadoras; assim, procurou-se controlar esse possível efeito da maturidade.

Em relação às vivências acadêmicas, a MANCOVA indicou efeitos significativos para sexo (Traço de Pillai $=0,102, p<0,001)$ e etapa (Traço de Pillai $=$ $0,056, p<0,001)$, mas não para a interação entre esses fatores (Traço de Pillai $=0,014, p=0,332$ ). Diferenças específicas nas subescalas do instrumento foram exploradas por meio de testes F de seguimento. Observaram-se diferenças estatisticamente significativas entre os sexos nas subescalas QVA-r Pessoal (homens com escores superiores aos das mulheres) e QVA-r Estudo (mulheres com escores superiores aos dos homens). Já na comparação entre calouros e formandos, foram verificadas diferenças estatisticamente significativas nas subescalas QVA-r Pessoal e QVA-r Institucional (em ambos os casos os calouros obtiveram escores superiores aos formandos).

Em relação ao coping, a MANCOVA indicou efeito significativo para sexo (Traço de Pillai $=0,115, p<$ 0,001 ), mas não para etapa (Traço de Pillai $=0,015, p$ $<0,201)$ e interação entre sexo e etapa (Traço de Pillai $=0,012, p=0,305)$. Novamente, diferenças específicas nas subescalas do instrumento foram exploradas por meio de testes $\mathrm{F}$ de seguimento. Observaram-se diferenças estatisticamente significativas entre os sexos nas subescalas EMEP-Emoção, EMEP-Religião/ Pensamento Fantasioso e EMEP-Suporte Social, com

Tabela 2

Regressão Linear Múltipla Predizendo Adaptação Acadêmica

\begin{tabular}{lccc} 
& Beta & $\mathrm{t}$ & $\mathrm{p}$ \\
\hline EMEP-Problema & 0,39 & 8,70 & $<0,001$ \\
EMEP-Emoção & $-0,29$ & $-6,22$ & $<0,001$ \\
EMEP-Religião/pens. fantasioso & $-0,07$ & $-1,39$ & 0,167 \\
EMEP-Suporte social & 0,13 & 2,67 & 0,008 \\
\hline
\end{tabular}

Psico-USF, Bragança Paulista, v. 20, n. 3, p. 421-432, set./ dez. 2015 
as mulheres apresentado escores mais altos do que os homens. Embora globalmente o efeito da etapa não tenha sido significativo, a comparação isolada de calouros e formandos mostrou um efeito significativo para EMEP-Problema, com os calouros apresentando escores mais elevados. Uma vez que essa diferença foi muito pequena $(3,68$ vs 3,63$)$, e análise global não indicou efeito da variável etapa, ela foi ignorada por não ser considerada relevante.

Além desses aspectos, pode-se constatar que, independentemente do grupo, as estratégias focadas no problema foram as mais utilizadas tanto por homens $(M=3,70)$ e mulheres $(M=3,64)$, quanto pelo grupo de calouros $(M=3,68)$ e formandos $(M=3,63)$. Já as estratégias baseadas na emoção foram as menos utilizadas por homens $(M=2,45)$ e mulheres $(M=2,58)$, calouros $(M=2,53)$ e formandos $(M=2,54)$.

\section{Discussão}

No presente estudo buscou-se investigar as possíveis relações entre estratégias de coping e o processo de adaptação acadêmica em estudantes universitários. Além disso, procurou-se verificar se as questões de gênero, ou o fato do aluno estar no primeiro ou último ano de seu curso, teriam relação com a escolha de determinadas estratégias e nos níveis de adaptação ao ensino superior. Com o intuito de abordar esses pontos, em um primeiro momento serão examinadas as principais correlações observadas na amostra. A seguir, serão discutidos alguns elementos da análise de regressão. Por fim, serão consideradas as principais médias obtidas pelos grupos.

As análises iniciais indicaram importantes correlações entre as variáveis coping e adaptação acadêmica. Entre os universitários, observou-se que o uso de estratégias focadas no problema constituiu-se em um preditor positivo da adaptação ao ensino superior. Estudos nacionais (Carlotto, Câmara, Otto, \& Kauffmann, 2010; Santos \& Alves Junior; 2007) e internacionais (Cohen, Ben-Zur, \& Rosenfeld, 2008; Gan, $\mathrm{Hu}$, \& Zhang, 2010) têm verificado que estratégias baseadas no confronto e na resolução de problemas podem colaborar para o manejo mais adequado dos estressores. Consequentemente, indivíduos com níveis reduzidos de estresse tendem a apresentar um processo de adaptação mais satisfatório (Crockett et al., 2007). Na amostra analisada, observou-se que o coping, baseado no problema, esteve mais relacionado com as dimensões Estudo, Institucional e Carreira do QVAr. Características próprias dessas estratégias, como a aceitação de responsabilidade, a resolução planejada de problemas e a proatividade (Lent et al., 2002), podem ter contribuído para essa relação. Provavelmente, a presença dessas estratégias tenha colaborado tanto para um maior desenvolvimento de hábitos e técnicas de estudo, quanto para um envolvimento mais significativo com questões estruturais e funcionais da instituição frequentada (Misra \& Mckean, 2000). Do mesmo modo, o enfrentamento dos desafios com foco no problema possibilita um melhor manejo das situações estressoras típicas do ambiente universitário, fazendo com que

Tabela 3

Comparações de Médias entre Homens e Mulheres, Calouros e Formandos

\begin{tabular}{|c|c|c|c|c|c|c|c|c|c|c|}
\hline & \multicolumn{2}{|c|}{ Homens } & \multicolumn{2}{|c|}{ Mulheres } & \multirow[b]{2}{*}{$p$} & \multicolumn{2}{|c|}{ Calouros } & \multicolumn{2}{|c|}{ Formandos } & \multirow[b]{2}{*}{$p$} \\
\hline & $\mathrm{M}$ & $\mathrm{DP}$ & $\mathrm{M}$ & $\mathrm{DP}$ & & $\mathrm{M}$ & $\mathrm{DP}$ & $\mathrm{M}$ & $\mathrm{DP}$ & \\
\hline QVA-r-Pessoal & 3,83 & 0,63 & 3,52 & 0,72 & $<0,001$ & 3,69 & 0,65 & 3,56 & 0,76 & 0,041 \\
\hline QVA-r-Interpessoal & 3,81 & 0,63 & 3,86 & 0,60 & 0,749 & 3,83 & 0,62 & 3,85 & 0,60 & 0,187 \\
\hline QVA-r-Carreira & 3,99 & 0,61 & 3,93 & 0,69 & 0,482 & 3,97 & 0,67 & 3,92 & 0,65 & 0,244 \\
\hline QVA-r-Estudo & 3,48 & 0,60 & 3,70 & 0,57 & 0,001 & 3,68 & 0,58 & 3,54 & 0,59 & 0,056 \\
\hline QVA-r-Institucional & 3,95 & 0,54 & 4,00 & 0,56 & 0,743 & 4,08 &, 54 & 3,84 &, 54 & 0,000 \\
\hline EMEP-Problema & 3,70 & 0,51 & 3,64 & 0,47 & 0,467 & 3,68 & 0,46 & 3,63 & 0,52 & 0,044 \\
\hline EMEP-Emoção & 2,45 & 0,56 & 2,58 & 0,57 & 0,036 & 2,53 & 0,55 & 2,54 & 0,59 & 0,487 \\
\hline EMEP-Religião/fant. & 2,68 & 0,70 & 3,13 & 0,73 & $<0,001$ & 2,97 & 0,75 & 2,94 & 0,75 & 0,703 \\
\hline EMEP-Suporte social & 3,03 & 0,82 & 3,40 & 0,91 & $<0,001$ & 3,25 & 0,91 & 3,28 & 0,88 & 0,533 \\
\hline
\end{tabular}

Nota. QVA-r = Questionário de Vivências Acadêmicas-revisado, EMEP = Escala Modos de Enfrentamento de Problemas. 
os estudantes que usam esse tipo de enfrentamento sintam-se mais identificados com seus cursos e escolhas profissionais à medida que se percebem capazes de superar as dificuldades colocadas pela vida acadêmica.

Em contrapartida, verificou-se que estratégias focadas na emoção estiveram correlacionadas negativamente com o processo de adaptação acadêmica, em especial, na dimensão que trata do ajustamento pessoal. Condutas típicas dessa categoria, como a negação, a fuga de problemas e o distanciamento, costumam ser relativamente eficazes em uma fase inicial do confronto com o evento estressor. No entanto, a médio e longo prazo tendem a interferir negativamente em aspectos relacionados à adaptação e à saúde (Dyson \& Renk, 2006). No presente estudo, percebeu-se que os estudantes que utilizavam o coping focado na emoção apresentavam índices consideravelmente mais reduzidos na dimensão Pessoal do QVA-r. É possível supor que, direta ou indiretamente, o uso dessas estratégias possa afetar de forma negativa aspectos pessoais relacionados ao bem-estar físico e psicológico, podendo contribuir, em alguns casos, para o desenvolvimento de quadros ansiosos e/ou depressivos (Crockett et al. 2007).

Por sua vez, as estratégias baseadas no suporte social apresentaram algumas especificidades. De forma geral, o uso dessas estratégias correlacionou-se de forma positiva com o processo de adaptação acadêmica, em especial com a dimensão Interpessoal das vivências acadêmicas, embora as magnitudes das correlações tenham sido bem baixas. Essa relação positiva era esperada, uma vez que os relacionamentos estabelecidos com amigos, cônjuges, pais, professores e funcionários costumam atuar como facilitadores da integração acadêmica (Costa \& Leal, 2006). No entanto, entre os acadêmicos avaliados, pôde-se verificar que altos escores de suporte social estiveram associados com baixos escores na dimensão Pessoal do QVA-r. Essa relação negativa, contudo, não chega a surpreender. Em alguns casos, observa-se que a procura frequente de suporte no outro pode ser resultado de uma inabilidade em lidar com os estressores de forma individual. Essa falta de capacidade individual de enfrentamento, por sua vez, pode levar ao surgimento de comportamentos inseguros, ansioso, passivos e dependentes. Assim, é compreensível que, frente a um sentimento de baixo bem-estar físico e psicológico, os estudantes busquem apoio no outro quando confrontados com situações estressoras (Cabral \& Matos, 2010).

As correlações entre a subescala Práticas religiosas/Pensamento fantasioso e as dimensões do QVA-r também apresentaram algumas particularidades. Em sua maior parte, essas estratégias associaram-se de forma negativa com o processo de adaptação, com destaque para a dimensão Pessoal. Esse resultado chama a atenção, pois, no campo da saúde, é possível encontrar um número significativo de estudos que têm apontado o potencial adaptativo do coping religioso (Pargament, Smith, Koenig, \& Perez, 1998; Faria \& Seidl, 2006; Simoni, Martone, \& Kerwin, 2002). Talvez, aspectos referentes ao instrumento utilizado para mensurar as práticas religiosas possam explicar, em parte, os resultados obtidos. Observa-se que boa parte dos itens que compuseram essa subescala expressa em seu conteúdo uma postura de esquiva ou de negação diante da situação estressora (pensamento fantasioso), e não necessariamente uma atitude de fé e esperança, que poderia estar associada com melhor ajustamento. Esses aspectos ficam evidentes nos itens 6 ("Espero que um milagre aconteça") e 41 ("Eu desejaria poder mudar o que aconteceu comigo"). Além disso, cabe observar que essa subescala apresentou estimativa de fidedignidade ligeiramente abaixo do desejável. Esse fato sugere que a medida pode não mensurar adequadamente o que se propõe (Alchieri, 2010). Salienta-se que as estratégias baseadas nas práticas religiosas/pensamento fantasioso mostraram-se adaptativas apenas em relação à dimensão Estudo do QVA-r.

$\mathrm{Na}$ análise de regressão, pode-se confirmar boa parte desses achados. Observou-se que as quatro subescalas de coping contribuíram para a predição do índice geral de vivências acadêmicas (adaptação acadêmica). Nessa análise, constatou-se que as estratégias focadas no problema e no suporte social relacionaram-se positivamente com a adaptação acadêmica; em contrapartida, as estratégias focadas na emoção e nas práticas religiosas correlacionaram-se negativamente com o processo de ajustamento ao ensino superior. Entre as quatro subescalas de coping avaliadas, verificou-se que as estratégias focadas no problema foram as mais relevantes para explicar a adaptação. De maneira geral, esses resultados estão de acordo com os estudos da área (Calais, Andrade, \& Lipp, 2003; Crockett et al. 2007; Lent et al., 2002).

Além das análises de correlação e regressão, buscou-se investigar se as questões de gênero, ou o fato do aluno estar no primeiro ou último ano de seu curso influenciariam na escolha de determinadas estratégias e nos níveis de adaptação ao ensino superior. De maneira geral, observou-se, na amostra uma predominância de estratégias focadas no problema e no suporte social. Provavelmente o uso frequente dessas estratégias, 
caracterizadas como adaptativas (Johnson, Gans, Kerr, \& LaValle, 2010), possa ter colaborado para os escores relativamente elevados obtidos no QVA-r.

Quanto às diferenças de gênero, constatou-se que estudantes do sexo feminino obtiveram médias mais elevadas do que os homens nas subescalas Suporte social, Emoção e Práticas religiosas/pensamento fantasioso. Em boa parte das pesquisas da área, tem-se observado que as mulheres utilizam com mais frequência as estratégias baseadas no suporte social (Costa \& Leal, 2006), nas práticas religiosas (Seidl et al., 2001) e nas emoções (Matud, 2004). Supõe-se que aspectos relacionados à socialização de ambos os sexos possam ter contribuído para essas diferenças. Observa-se que, desde a infância, as mulheres costumam ser mais estimuladas a utilizarem estratégias pró-sociais, como a busca de apoio diante de problemas e a manutenção de fortes relações com a família (Calais, 2003). Além disso, acredita-se que, no sexo feminino, possa existir uma motivação cultural para perceber e expressar com maior facilidade as emoções (Antoniazzi, Dell'Aglio, \& Bandeira, 1998; Calais, 2003).

Em relação às dimensões do QVA-r, também se pode constatar algumas diferenças importantes quanto ao gênero. Observou-se que as estudantes do sexo feminino apresentaram níveis mais elevados na dimensão Estudo. Nesse caso, é plausível afirmar que as mulheres dessa amostra perceberam-se mais competentes em questões relacionadas aos hábitos de estudo, gestão do tempo e estratégias de aprendizagem (Schleich, 2006). Já, nos homens, pode-se verificar a presença de escores superiores na dimensão Pessoal. Esses índices permitem afirmar que os estudantes do sexo masculino perceberam-se com melhores condições físicas e psicológicas do que as mulheres. Escores elevados na dimensão Pessoal podem estar associados a maiores níveis de autonomia e otimismo (Bariani, 2005).

Entre alunos ingressantes e concluintes não se observaram diferenças significativas quanto ao uso de estratégias de coping. $\mathrm{Na}$ amostra analisada, constatou-se que tanto calouros quanto formandos utilizaram com mais frequência estratégias focadas no problema e no suporte social. Esse resultado chama a atenção, pois esperava-se que a menor experiência dos calouros frente aos desafios do contexto acadêmico poderia levá-los a usar com mais intensidade estratégias menos adaptativas, quando comparados com os formandos (Toray \& Cooley, 1998).

Nas dimensões do QVA-r, constataram-se diferenças significativas entre ingressantes e concluintes nas subescalas Institucional e Pessoal. $\mathrm{Na}$ amostra analisada, os calouros apresentaram níveis mais elevados em ambas as dimensões. Ao ingressar na universidade, o estudante ainda carece de um conhecimento mais amplo sobre o significado de estar no ensino superior. Muitas vezes, o primeiro ano constitui-se como um período de idealização do ambiente universitário. Em boa parte dos casos, observa-se que o estudante ainda não desenvolveu uma visão crítica sobre os pontos fortes e fracos da instituição frequentada. Supõe-se que elementos relacionados a essa idealização possam ter contribuído para que os calouros obtivessem escores superiores nessa dimensão (Almeida \& Soares, 2003). Por outro lado, verifica-se que os alunos que estão concluindo seus cursos, em função das experiências acumuladas ao longo da graduação, costumam apresentar uma visão mais crítica sobre vários aspectos institucionais (estrutura, serviços, intenção em permanecer e compromisso com a universidade frequentada). Possivelmente, esse fato possa ter contribuído para os níveis mais reduzidos apresentados pelos formandos nessa dimensão (Igue et al., 2008).

Em relação ao escore mais baixo dos formandos na dimensão Pessoal, deve-se considerar que essa diferença foi menos expressiva. De toda forma, é possível supor que esses estudantes, por estarem se aproximando de uma transição de carreira (escola-trabalho), pudessem estar experimentando preocupação em relação ao futuro, o que poderia se refletir em mal-estar físico e mesmo psicológico.

Por fim, ainda merece destaque o fato de não terem sido encontradas diferenças significativas entre calouros e formandos nas demais dimensões do QVA-r. $\mathrm{Na}$ medida em que o primeiro ano de graduação é caracterizado por um número significativo de estressores (Guerreiro-Casanova \& Polydoro, 2010), esperava-se observar em ingressantes níveis inferiores de adaptação acadêmica (Almeida \& Soares, 2003), fato que não foi constatado. Talvez, o uso mais frequente de estratégias de enfrentamento focadas no problema e no suporte social pelos calouros possa explicar, em parte, os bons índices de adaptação observados nesse grupo.

\section{Considerações Finais}

De modo geral, os resultados, nesta pesquisa, confirmam alguns achados da literatura e apontam para variáveis que merecem uma maior atenção em futuros estudos sobre a adaptação à vida universitária. Pode-se perceber que as estratégias focadas no 
problema e no suporte social correlacionaram-se positivamente com o processo de adaptação acadêmica. Por outro lado, as estratégias focadas na emoção estiveram relacionadas negativamente com o processo de ajustamento ao ensino superior. Esses dados estão de acordo com a maior parte dos resultados da literatura nacional e internacional. No entanto, nas análises realizadas, chama a atenção o fato das estratégias focadas nas práticas religiosas terem se mostrado relacionadas com menor adaptação. Talvez a realização de estudos com instrumentos mais adaptados à realidade dos universitários possa contribuir para a apresentação de resultados mais positivos em relação ao uso das práticas religiosas e espirituais.

$\mathrm{Na}$ amostra avaliada, pode-se observar que as mulheres, comparativamente aos homens, apresentaram escores significativamente mais elevados nas subescalas Emoção, Práticas religiosas/pensamento fantasioso e Suporte social. De maneira geral, esse resultado está de acordo com várias publicações da área. No entanto, quando essas comparações são realizadas entre alunos ingressantes e concluintes, alguns pontos chamam a atenção. Surpreende o fato de não se observar diferenças significativas no uso de estratégias de enfrentamento entre os alunos de primeiro e último ano. Nesse caso, sugere-se o desenvolvimento de pesquisas que investiguem os motivos dessa ausência de diferenças em relação ao uso do coping.

Entende-se que o aprofundamento das investigações acerca das variáveis que afetam a adaptação acadêmica possa constituir-se em uma ferramenta importante para gestores educacionais. Informações do gênero podem contribuir para a implementação de programas institucionais que auxiliem no desenvolvimento de estratégias de enfrentamento adaptativas entre os estudantes. Especificamente, acredita-se que a criação de intervenções favorecedoras do coping focado no problema e no suporte social possa colaborar para um melhor manejo das situações estressantes vivenciadas pelos acadêmicos.

Apesar dos resultados observados, destacam-se duas limitações importantes do estudo. A primeira delas diz respeito às especificidades dos instrumentos utilizados na pesquisa. As cinco dimensões do QVA-r, embora abrangentes, com certeza não cobrem todo o espectro de experiências relacionadas à vida universitária. Da mesma forma, as quatro subescalas propostas pelo EMEP não conseguem contemplar de forma integral o espectro do coping. A segunda limitação refere-se ao caráter transversal do estudo. Para que se possa determinar com mais precisão as relações temporais entre essas variáveis, sugere-se a realização de pesquisas longitudinais.

Cabe ressaltar que mais pesquisas sobre o assunto são necessárias. A investigação da relação entre estratégias de enfrentamento e outras variáveis como bem-estar, resiliência, características de personalidade e lócus de controle, entre outros, poderiam ser importantes para consolidar as evidências sobre o papel adaptativo do coping junto ao processo de integração acadêmica. Além disso, a abordagem do tema por meio de outras perspectivas, de modo que se possa ampliar a compreensão do fenômeno da adaptação à universidade no contexto brasileiro, pode ser igualmente útil.

\section{Referências}

Adolhe, R., Guido, L. A., \& Bianchi, E. R. F. (2009). Stress e coping no período perioperatório de câncer de mama. Revista da Escola de Enfermagem da USP, 43(3), 711-720. doi: 10.1590/S0080-62342009000300030

Alchieri, J. C. (2010). Análise dos dados demográficos das normas brasileiras de instrumentos psicológicos empregados na avaliação da personalidade. Revista Psicologia e Saúde, 2(1), 56-63. Recuperado de http://www.gpec.ucdb.br/pssa/index.php/pssa/ article/view/36

Almeida, L. S., Guisande, M. A., Soares, A. P., \& Saavedra, L. (2006). Acesso e sucesso no Ensino Superior em Portugal: Questões de género, origem sociocultural e percurso académico dos alunos. Psicologia: Reflexão e Crítica, 19(3), 507-514. doi: 10.1590/S0102-79722006000300020

Almeida, L. S., \& Soares, A. P. (2003). Os estudantes universitários: Sucesso escolar e desenvolvimento psicossocial. In E. Mercuri \& S. A. J. Polydoro (Eds.), Estudante universitário: Características e experiências de formação (pp. 15-40). Taubaté, SP: Cabral Universitária.

Antoniazzi, A. S., Dell'Aglio, D. D., \& Bandeira, D. R. (1998). O conceito de coping: Uma revisão teórica. Estudos de Psicologia, 3(2), 273-294. doi: 10.1590/ S1413-294X1998000200006

Aspinwall, L. G., \& Taylor, S. E. (1992). Modeling cognitive adaptation: A longitudinal investigation of the impact of individual differences and coping on college judgment and performance. Journal 
of Personality and Social Psychology, 63(6), 989-1003. Recuperado de http://www.ncbi.nlm.nih.gov/ pubmed/1460565

Bardagi, M. P., \& Hutz, C. S. (2011). Eventos estressores no contexto acadêmico: Uma breve revisão da literatura. Interação em Psicologia, 15(1), 111-119. doi: http://dx.doi.org/10.5380/psi.v15i1.17085

Bariani, I. C. D. (2005). Vivência acadêmica de universitários ingressantes. Relatório técnico de pesquisa. Campinas: Faculdade de Psicologia, PUC.

Cohen, M., Ben-Zur, H., \& Rosenfeld, M. J. (2008). Sense of coherence, coping strategies, and test anxiety as predictors of test performance among college students. International Journal of Stress Management, 15(3), 289-303. doi: 10.1037/1072-5245.15.3.289

Cabral, J., \& Matos, P. M. (2010). Preditores da adaptação à universidade: $O$ papel da vinculação, desenvolvimento psicossocial e coping. Psychologica, 1(52), 55-77. doi: 10.14195/1647-8606_52-1_4

Calais, S. L., Andrade, L. M. B., \& Lipp, M. E. N. (2003). Gender and schooling differences in stress symptoms in young adults. Psicologia: Reflexão e Crítica 16(2), 257-263. doi: 10.1590/S0102-79722003000200005

Carlotto, M. S., Câmara, S. G., Otto, F., \& Kauffmann, P. (2010). Síndrome de burnout e coping em estudantes de psicologia. Boletim de Psicologia, 59(131), 167-178. Recuperado de http:// pepsic.bvsalud.org/scielo.php?script=sci_arttext\& pid $=$ S0006-59432009000200004

Coelho, J. A. P. M., Albuquerque, F. J. B., Martins, C. R., D’Albuquerque, H. B., \& Neves, M. T. S. (2008). Coping em jovens frente à expectativa de inserção ocupacional e indicadores de depressão. Psicologia: Teoria e Pesquisa, 24(4), 527-534. Recuperado de http://www.scielo.br/pdf/ptp/v24n4/17.pdf

Costa, E. S., \& Leal, I. P. (2006). Estratégias de coping em estudantes do ensino superior. Análise Psicológica, 2(24), 189-199. Recuperado de http://www.scielo. mec.pt/pdf/aps/v24n2/v24n2a06.pdf

Crockett, L. J., Iturbide, M. I., Torres-Stone, R. A., McGinley, M., Raffaelli, M., \& Carlo, G. (2007). Acculturative stress, social support, and coping: Relations to psychological adjustment among Mexican American college students. Cultural Diversity and Ethnic Minority Psychology, 13(4), 347-355. doi: 10.1037/1099-9809.13.4.347
Dela Coleta, A. S. M., \& Dela Coleta, M. F. (2008). Fatores de estresse ocupacional e coping entre policiais civis. Psico-USF, 13(1), 59-68. Recuperado de http://www.scielo.br/pdf/pusf/v13n1/ v13n1a08.pdf

Diniz, A. M., \& Almeida, L. S. (2006). Adaptação à universidade em estudantes de primeiro ano: Estudo diacrónico da interação entre o relacionamento com pares, o bem-estar pessoal e o equilíbrio emocional. Análise Psicológica, 24(1), 29-38. Recuperado de http://hdl.handle.net/10400.12/154

Dyson, R., \& Renk K. (2006). Freshmen adaptation to university life: Depressive symptoms, stress, and coping. Journal of Clinical Psychology, 62(10), 12311244. Recuperado de http://www.ncbi.nlm.nih. gov/pubmed/16810671

Faria, J. B., \& Seidl, E. M. F. (2006). Religiosidade, enfrentamento e bem-estar subjetivo em pessoas vivendo com HIV/AIDS. Psicologia em Estudo, 11(1), 155-164. Recuperado de http://www.scielo. br/pdf/pe/v11n1/v11n1a18

Feenstra, S. J., Banyard, L. V., Rines, E. N., \& Hopkins, R. K. (2001). First-year students' adaptation to college: The role of family variables and individual coping. Journal of College Student Development, 42(2), 106113. Recuperado de http://www.researchgate.net/ publication/234745454_First-Year_Students'_Adaptation_to_College_The_Role_of_Family_Variables_and_Individual_Coping

Gan, Y., Hu, Y., \& Zang, Y. (2010). Proactive and preventive coping in adjustment to college. The Psychological Record, 60(4), 643-658. Recuperado de http://www.psy.pku.edu.cn/Upload/ pubs/2010090300001.pdf

Gimenes, M. G. G., \& Queiroz, B. (1997). As diferentes fases de enfrentamento durante o primeiro ano após a mastectomia. In M. G. G. Gimenes \& M. H. Fávero (Ed.). A mulher e o câncer (pp. 171-195). Campinas: Editorial Psy.

Granado, J. I. F., Santos, A. A. A., Almeida, L. S, Soares, A. P., \& Guisande, M. A. (2005). Integração acadêmica de estudantes universitários: Contributos para a adaptação e validação do QVA-r no Brasil. Psicologia e Educação, 1, 33-43. Recuperado de http://hdl.handle.net/1822/12089

Guerreiro-Casanova, D. C., \& Polydoro, S. A. J. (2010). Integração ao ensino superior: Relações ao longo 
do primeiro ano de graduação. Psicologia: Ensino \& Formação, 1(2), 85-96. Recuperado de http://pepsic.bvsalud.org/pdf/pef/v1n2/v1n2a08.pdf

Igue, E. A., Bariani, I. C. D., \& Milanesi, P. V. B. (2008). Vivência acadêmica e expectativas de universitários ingressantes e concluintes. Psico-USF, 13(2), 155164. doi: 10.1590/S1413-82712008000200003

Johnson, V. K., Gans, S. E., \& Kerr, S., \& LaValle, W. (2010) Managing the transition to college: Family functioning, emotion coping, and adjustment in emerging adulthood. Journal of College Student Development, 51(6), 607-621. doi: 10.1353/csd.2010.0022

Lazarus, R. S., \& Folkman, S. (1984). Stress, appraisal, and coping. New York, United States: Springer.

Lent, R. W., Brown, S. D., Talleyrand, R., McPartland, E. B., Davis, T., Chopra, S. B., Suthakaran, V., \& Chai, C. (2002). Career choice barriers, supports, and coping strategies: College students' experiences. Journal of Vocational Behavior, 60(1), 61-72. doi:10.1006/jvbe.2001.1814

Matud, M. P. (2004). Gender differences in stress and coping styles. Personality and Individual Differences, 37(7), 1401-1415. doi:10.1016/j.paid.2004.01.010

Misra, R., \& Mckean, M. (2000). College student's academic stress and its relation to their anxiety, time management, and leisure satisfaction. American Journal of Health Studies, 16(1), 41-51. Recuperado de http://www.biomedsearch.com/article/College-students-academic-stress-its/65640245.html

Neme, C. M. B., \& Lipp, M. E. N. (2010). Estresse psicológico e enfrentamento em mulheres com e sem câncer. Psicologia: Teoria e Pesquisa, 26(3), 475-483. doi: 10.1590/S0102-37722010000300010

Pargament, K. I., Smith, B. W., Koenig, H. G., \& Perez, L.M. (1998). Patterns of positive and negative religious coping with major life stressors. Journal for the Scientific Study of Religion, 37(4), 710-724. doi: $10.2307 / 1388152$

Pascarella, E. T., \& Terenzini, P. T. (2005). How college affects students. Vol. 2: A third decade of research. San Francisco: Jossey-Bass.

Santos, A. F., \& Alves Júnior, A. (2007). Estresse e estratégias de enfrentamento em mestrandos de ciências da saúde. Psicologia: Reflexão e Crítica, 20(1), 104-113. doi: 10.1590/S0102-79722007000100014.
Schleich, A. L. R. (2006). Integração na educação superior e satisfação acadêmica de estudantes ingressantes e concluintes (Dissertação de mestrado). Campinas: Universidade Estadual de Campinas.

Seidl, E. M. F., Tróccoli, B. T. \& Zannon, C. M. L. C. (2001). Análise fatorial de uma medida de estratégias de enfrentamento. Psicologia: Teoria e Pesquisa, 17(3), 225-234. doi: 10.1590/S0102-37722001000300004

Simoni J. M., Martone M. G., \& Kerwin J. F. (2002). Spirituality and psychological adaptation among women with HIV/AIDS: Implications for counseling. Journal of Counseling Psychology. 49(2), 139-147. Recuperado de http://psycnet.apa.org/index. cfm? $\mathrm{fa}=$ buy.option ToBuy\&id $=2002-12450-001$

Soares, A. B., Poubel, L. N., \& Mello, T. V. S. (2009). Habilidades sociais e adaptação acadêmica: Um estudo comparativo em instituições de ensino público e privado. Aletheia, 29, 27-42. Recuperado de http:/ / pepsic.bvsalud.org/scielo.php?pid=S1413-03942009000100004\&script $=$ sci_arttext

Suehiro, A. C. B., Santos, A. A. A., Hatamoto, C. T., \& Cardoso, M. M. (2008). Vulnerabilidade ao estresse e satisfação no trabalho em profissionais do Programa de Saúde da Família. Boletim de Psicologia, 58(129), 205-218. Recuperado de http://ltc-ead.nutes.ufrj. br/constructore/objetos/SUEHIRO_A.C.B._ et.alli_Vulnerabilidade.ao.estresse.e.satisfacao. no.trabalho.em.profissionais.do.Programa. de.Saude.da.Familia\%5B1\%5D.pdf

Swenson, L. M., Nordstrom, A., \& Hiester, M. (2008). The role of peer relationships in adjustment to college. Journal of College Student Development, 49(6), 551-567. doi: 10.1353/csd.0.0038

Teixeira, M. A. P., Dias, A. C. G., Wottrich, S. H., \& Oliveira, A. M. (2008). Adaptação à universidade em jovens calouros. Revista Semestral da Associação Brasileira de Psicologia Escolar e Educacional, 12(1), 185-202. Recuperado de http://www.scielo.br/ pdf/pee/v12n1/v12n1a13.pdf

Toray, T., \& Cooley, E. (1998).Coping in women college student's: The influence of experience. Journal of College Student Development, 39(3), 291-295. Recuperado de http:// eric.ed.gov/?id=EJ569838

Recebido: 06/12/2013

Primeira reformulação: 26/01/2015

Aprovado: 09/02/2015 
Sobre os autores:

Rodrigo Carvalho Carlotto é graduado em Psicologia pela Universidade Federal de Santa Maria (2007), mestre em Psicologia pela Universidade Federal de Santa Maria (2013) e, atualmente, é psicólogo no Instituto Federal Farroupilha - Campus Júlio de Castilhos.

E-mail: rodrigocarlotto@yahoo.com.br

Marco Antônio Pereira Teixeira é professor adjunto do Programa de Pós-Graduação em Psicologia, pela Universidade Federal do Rio Grande do Sul.

E-mail:mapteixeira@yahoo.com.br

Ana Cristina Garcia Dias é graduada (1995) e licenciada (1997) em Psicologia pela Universidade Federal do Rio Grande do Sul/UFRGS (1995), mestre (1998) e doutora em Psicologia (2003) pela Universidade de São Paulo e, atualmente, professora Adjunta da Universidade Federal do Rio Grande do Sul. Área de concentração: Psicologia Escolar e do Desenvolvimento Humano.

E-mail: anacristinagarciadias@gmail.com

\section{Contato com os autores:}

Rodrigo Carvalho Carlotto

E-mail: rodrigocarlotto@yahoo.com.br

Rua Alfredo Tonetto, 194, Bairro Camobi, Santa Maria/RS

CEP: 97110-802 